\title{
Myocardial Perfusion Imaging Versus CT Coronary Angiography: When to Use Which?
}

\author{
Balaji Tamarappoo and Rory Hachamovitch \\ Section of Cardiovascular Imaging, Department of Cardiovascular Medicine, Cleveland Clinic, Cleveland, Ohio
}

\begin{abstract}
Learning Objectives: On successful completion of this activity, participants should be able to describe (1) the various types of noninvasive cardiac imaging tests and how the results of each are utilized; (2) recent advances in CT coronary angiography, PET myocardial perfusion imaging, and SPECT myocardial perfusion imaging of coronary artery disease; and (3) the clinical use of anatomic versus physiologic tests in symptomatic patients with known or suspected coronary artery disease.
\end{abstract}

Financial Disclosure: The authors of this article have indicated no relevant relationships that could be perceived as a real or apparent conflict of interest.

CME Credit: SNM is accredited by the Accreditation Council for Continuing Medical Education (ACCME) to sponsor continuing education for physicians. SNM designates each JNM continuing education article for a maximum of 1.0 AMA PRA Category 1 Credit. Physicians should claim only credit commensurate with the extent of their participation in the activity.

For CE credit, participants can access this activity through the SNM Web site (http://www.snm.org/ce_online) through June 2012.

Both anatomy- and physiology-based approaches to patient management have advantages and limitations. Compared with the latter, the former has a superior ability to exclude disease and does not miss high-risk coronary artery disease (CAD). However, it is limited by a possibility of overestimating the severity of CAD and of potentially failing to determine which posttest therapeutic approach optimizes treatment benefit. On the other hand, although a physiology-based approach could potentially identify optimal therapeutic strategies, the possibility of both false-positive and false-negative findings is a concern. This review incorporates some of the more recent advances in CT coronary angiography and myocardial perfusion imaging (MPI), including PET MPI, into a discussion of anatomic versus physiologic imaging and provides our perspective on how an anatomy-based testing strategy centered in CT coronary angiography versus a physiology-based testing strategy with MPI may be clinically used for the evaluation of known or suspected CAD in symptomatic patients.

Key Words: CT coronary angiography; myocardial perfusion; review

J Nucl Med 2011; 52:1079-1086

DOI: 10.2967/jnumed.110.081133

$\mathbf{N}$ oninvasive cardiac imaging tests are often the first step in the assessment of coronary anatomy, myocardial perfusion, or left ventricular function in patients with known or

Received Jan. 28, 2011; revision accepted May 16, 2011.

For correspondence or reprints contact: Rory Hachamovitch, Section of Cardiovascular Imaging, Department of Cardiovascular Medicine, Cleveland Clinic, 9500 Euclid Ave., Desk J1-5, Cleveland, OH 44195.

E-mail: hachamr@ccf.org

COPYRIGHT @ 2011 by the Society of Nuclear Medicine, Inc. suspected coronary artery disease (CAD). The results of these tests are used for patient risk stratification, evaluation of myocardial ischemia as a cause of symptoms, and assessment of ongoing disease management. Stress myocardial perfusion imaging (MPI) with SPECT is a well-established modality capable of quantifying relative stress and rest myocardial perfusion and left ventricular function with established diagnostic accuracies and prognostic performance characteristics. More recently, perfusion imaging with PET has been shown to have similar diagnostic accuracy for detection of CAD, with the added advantage of enhanced image quality and the potential to quantify absolute myocardial blood flow. The introduction of noninvasive CT coronary angiography as part of routine patient care allowed anatomic characterization of coronary arteries with outstanding accuracy for detecting the presence of anatomic obstructions secondary to CAD. More recently, evaluation of the prognostic value of this test has been reported by several centers. Interestingly, the availability of CT coronary angiography has brought to the forefront the longstanding question of assessing CAD on the basis of anatomic criteria (CT coronary angiography) versus physiologic criteria (MPI). The role of coronary calcium score, CT coronary angiography, and MPI for the evaluation of asymptomatic intermediate- to high-risk patients and symptomatic patients has previously been discussed by Berman et al. in a comprehensive review (1). The goal of this current review is to incorporate some of the more recent advances in CT coronary angiography and MPI, including PET MPI, into this discussion of anatomic versus physiologic imaging and to provide our perspective on how an anatomy-based testing strategy centered in CT coronary angiography versus a physiologybased testing strategy with MPI may be clinically used for the evaluation of known or suspected CAD in symptomatic patients. 


\section{ANATOMY-BASED APPROACH: CT CORONARY ANGIOGRAPHY}

\section{Diagnostic Value}

A recent review of advanced cardiovascular imaging summarized the published studies examining the diagnostic accuracy of CT coronary angiography. The weighted sensitivity and specificity of these studies were relatively high (per patient, 94\% and 77\%, respectively; per segment, 83\% and $92 \%$, respectively) (2). Methodologically, these studies were based on the recruitment of stable patients referred for elective catheterization and for a research CT coronary angiography study. Not surprisingly, in light of this source of patient recruitment, the prevalence of CAD was relatively high $(62 \%)$, thus affecting both positive predictive value and negative predictive value (per patient, $84 \%$ and $87 \%$, respectively). CT coronary angiography is unlikely to be applied in practice to populations with this high a prevalence of CAD. If recalculated for a CAD prevalence of $15 \%$ (more likely to be seen in practice), the positive and negative predictive values become $46 \%$ and $98 \%$, respectively.

A more realistic assessment of CT coronary angiography was obtained by the ACCURACY trial (Assessment by Coronary Computed Tomographic Angiography of Individuals Undergoing Invasive Coronary Angiography), a prospective, multicenter study enrolling 230 patients with a low to intermediate pretest likelihood of CAD who underwent both CT coronary angiography and invasive coronary angiography (3). In that study, although observed sensitivity and specificity were very good to excellent $(93.8 \%$ and $81.8 \%$, respectively, for detecting stenosis $>70 \%$ ) and negative predictive value was outstanding $(98.8 \%)$, the positive predictive value of CT coronary angiography was only $47.6 \%$. This study illustrated that CT coronary angiography, although able to exclude obstructive CAD with confidence, misidentifies disease in approximately half of patients with positive test results. In patients with a CT coronary angiography-based stenosis of more than $50 \%$, there is still a wide margin of error in the grading of stenosis severity, reducing the accuracy of identifying individuals whose symptoms may be attributed to myocardial ischemia. In the nonacute setting, CT coronary angiography is extremely valuable in ruling out CAD but varies widely in the ability to identify the exact severity of luminal stenosis and, therefore, its hemodynamic relevance.

Subsequently, several studies have examined the relationship between assessment of coronary artery stenosis on CT coronary angiography and assessment of myocardial ischemia on SPECT MPI and PET MPI. These retrospective studies showed that only $30 \%-50 \%$ of all individuals with a maximal stenosis of more than $50 \%$ exhibited myocardial ischemia on MPI (4-7). In the subgroup of individuals with a maximal stenosis of $50 \%-75 \%$, the prevalence of ischemia was even lower, ranging from $16 \%$ to $20 \%$. A more recent study of 292 patients comparing luminal stenosis by $\mathrm{CT}$ coronary angiography and myocardial ischemia by SPECT MPI showed that at the extremes of luminal stenosis severity $(<50 \%$ [negative predictive value, $98 \%$ ] and $\geq 90 \%$ [positive predictive value, $74 \%$ ]), CT coronary angiography capably predicted the absence or presence of ischemia (8). In contrast, there was wide variability in the prevalence of ischemia among patients with a luminal stenosis severity of 50\%-90\%. All these studies demonstrated that the absence of plaque on CT coronary angiography can be an extremely useful finding in ruling out CAD in symptomatic patients; however, in almost 50\% of low- to intermediate-likelihood patients with stenosis greater than $50 \%$ on CT coronary angiography, a second noninvasive imaging test may be necessary to definitively diagnose ischemia as the cause of symptoms.

\section{Prognostic Value}

The prognostic literature supporting the use of CT coronary angiography has been greatly expanded over the past 5 years, and several important points emerge from the current literature. First, there is little doubt that a normal CT coronary angiography study, particularly in the absence of any evidence of atherosclerosis, is associated with both a very high negative predictive value for the occurrence of adverse events and a low likelihood of obstructive CAD. A recent metaanalysis of 18 studies evaluating 9,592 patients with a 20 -mo median follow-up revealed a $0.15 \% / y$ rate of death or myocardial infarction and a $0.17 \%$ rate of death, myocardial infarction, or revascularization (major adverse coronary event) (9). Thus, the risk of adverse cardiovascular events after a completely normal CT coronary angiography result is comparable to the baseline risk of a healthy patient. Further, significant risk stratification is achieved by the results of CT coronary angiography in that among the 2,772 patients with obstructive CAD, the authors reported annualized rates of $8.8 \%$ for a major adverse coronary event, $2.2 \%$ for death, and $2.1 \%$ for myocardial infarction. These results suggest an efficient stratification, with an abnormal study indicating a relative risk more than 40 times that of a normal study.

Further, as has been shown with MPI, the risk of adverse events is closely associated with the extent and severity of underlying CAD. In a study of 1,127 low- to intermediaterisk symptomatic patients referred for 16-slice $\mathrm{CT}$ coronary angiography for the diagnosis of stable chest pain syndrome, Min et al. demonstrated that during a 15-mo follow-up, the risk of death increased with the number of vessels that had atherosclerotic plaque and with the severity of stenosis. Further, risk was greatest in patients who had plaque in the left main coronary artery or the proximal left anterior descending coronary artery $(10)$.

To evaluate whether CT coronary angiography has incremental prognostic value over MPI, a recent study followed 541 patients at intermediate risk for CAD (defined as 15\%$85 \%$ ) who underwent both CT coronary angiography and SPECT MPI. Luminal stenosis greater than $50 \%$ was detected by CT coronary angiography in $31 \%$ of study 
patients, and abnormal myocardial perfusion (summed stress score $\geq 4$ ) was observed in $33 \%$. After adjustment for clinical risk factors, both obstructive plaque on CT coronary angiography and abnormal results on SPECT MPI were independent predictors of late events, and the combined use of the 2 modalities significantly enhanced the prediction of major adverse cardiac events, including all-cause mortality, revascularization, and infarction (11).

Unlike invasive coronary angiography, CT coronary angiography can assess atherosclerotic plaque location, morphology, and composition (2). It is likely that with time, the prognostic and therapeutic implications of this information will be used to further enhance prognostication and its application to clinical practice (12). Similarly, a normal MPI result, although excluding obstructive CAD, cannot determine whether the disease process has already started, thus differentiating between the need for primary versus secondary prevention (2). On the other hand, CT coronary angiography is similar to invasive coronary angiography, in that it has several significant limitations. Neither modality can reliably measure the functional significance of coronary stenosis (2). Also similar to invasive angiography, CT coronary angiography is ineffective in determining which individual plaque is likely to be the site of a future acute coronary event $(2,12)$.

\section{Posttest Management Strategy}

In Figure 1, we outline an anatomy-based posttest management strategy for a stable, symptomatic patient with an intermediate to high likelihood of $50 \%-85 \%$ (1). An anatomy-based approach in a patient with unstable angina would most likely comprise invasive coronary angiography. In patients with normal findings on CT coronary angiography, both obstructive CAD and atherosclerosis are effectively excluded, and the patient is thus a candidate for primary prevention. In patients showing nonobstructive lesions $(<50 \%$ stenosis) on CT coronary angiography, there is no need for further noninvasive assessment with SPECT MPI or for invasive assessment. However, this result still indicates a diagnosis of CAD, and secondary prevention would thus be the best subsequent course. In patients showing mild obstructive lesions (50\%-70\% stenosis) on CT coronary angiography, secondary prevention would still be the required clinical course. However, the presence of limiting symptoms, limited functional capacity, or other issues resulting in recurrent patient presentation would justify a noninvasive imaging strategy with SPECT MPI or PET MPI to rule out myocardial ischemia. Finally, in patients with results suggestive of high-risk CAD (left main lesion or proximal left anterior descending lesion) on CT coronary angiography, it is generally agreed that referral for invasive assessment in conjunction with secondary prevention measures is an appropriate clinical course. Many centers perform gated CT studies, thus permitting assessment of left ventricular ejection fraction and wall motion as part of routine studies. Patients found to have left ventricular dysfunction in conjunction with obstructive CAD may be considered candidates for a more aggressive course of treatment and are more likely to be referred for invasive assessment.

\section{IMAGING IN A POST-COURAGE WORLD}

The results of the COURAGE trial (Clinical Outcomes Utilizing Revascularization and Aggressive Drug Evaluation) indicated that survival benefit was not enhanced - nor quality of life improved-when percutaneous coronary intervention (PCI) was added to a strategy of aggressive medical therapy in patients with documented obstructive CAD (13). Although the implications of this trial for the use of revascularization procedures are well appreciated, the implications for the imaging community are often overlooked. In most patients currently referred for stress MPI or CT coronary angiography in the United States, the referral is for the purpose of assessing known or suspected CAD. If, as suggested by COURAGE, neither survival nor quality of life will benefit from revascularization, the routine use of noninvasive imaging to detect possible ischemia and identify possible revascularization candidates is unnecessary. In a future world shaped by COURAGE, the use of imaging in the assessment of ischemic heart disease will be limited to evaluation of those patients suspected of having left main CAD.

The COURAGE trial was performed on patients with documented obstructive CAD. Many of these patients may have had either limited or no inducible ischemia or extensive prior myocardial infarction. Although the COURAGE trial does suggest a lack of revascularization benefit in patients identified on anatomic grounds, data support a possible role for assessment of ischemia or flow reserve in the identification of patients who may benefit from revascularization.

The use of fractional flow reserve-defined as the ratio of pressure proximal and distal to a stenotic lesion during vasodilator-induced hyperemia - to measure the physiologic significance of anatomic lesions at the time of invasive angiographic studies has been shown to play a significant role in identifying revascularization candidates. In patients with a fractional flow reserve greater than 0.75 , PCI can be deferred without increased patient risk, despite the angiographic appearance of a significant stenosis (14). Furthermore, cardiac event rates during a 5-y follow-up were lower in patients with a fractional flow reserve greater than 0.75 without PCI than with PCI $(15,16)$. Even in the setting of left main $\mathrm{CAD}$, these investigators found that a fractional flow reserve greater than 0.75 was associated with an excellent 3-y survival rate and freedom from major adverse cardiovascular events (17). Conversely, event rates increased when lesions with a fractional flow reserve lower than 0.75 were not revascularized (18). Finally, FAME (Fractional Flow Reserve Versus Angiography for Multivessel Evaluation), a prospective multicenter randomized trial, compared a fractional flow reserve-guided versus angiography-based revascularization strategy in 1,005 patients with multivessel CAD (19). At $1 \mathrm{y}$, the fractional flow reserve-guided strategy reduced the rate of a composite 
FIGURE 1. Potential testing algorithm based on use of CT coronary angiography.

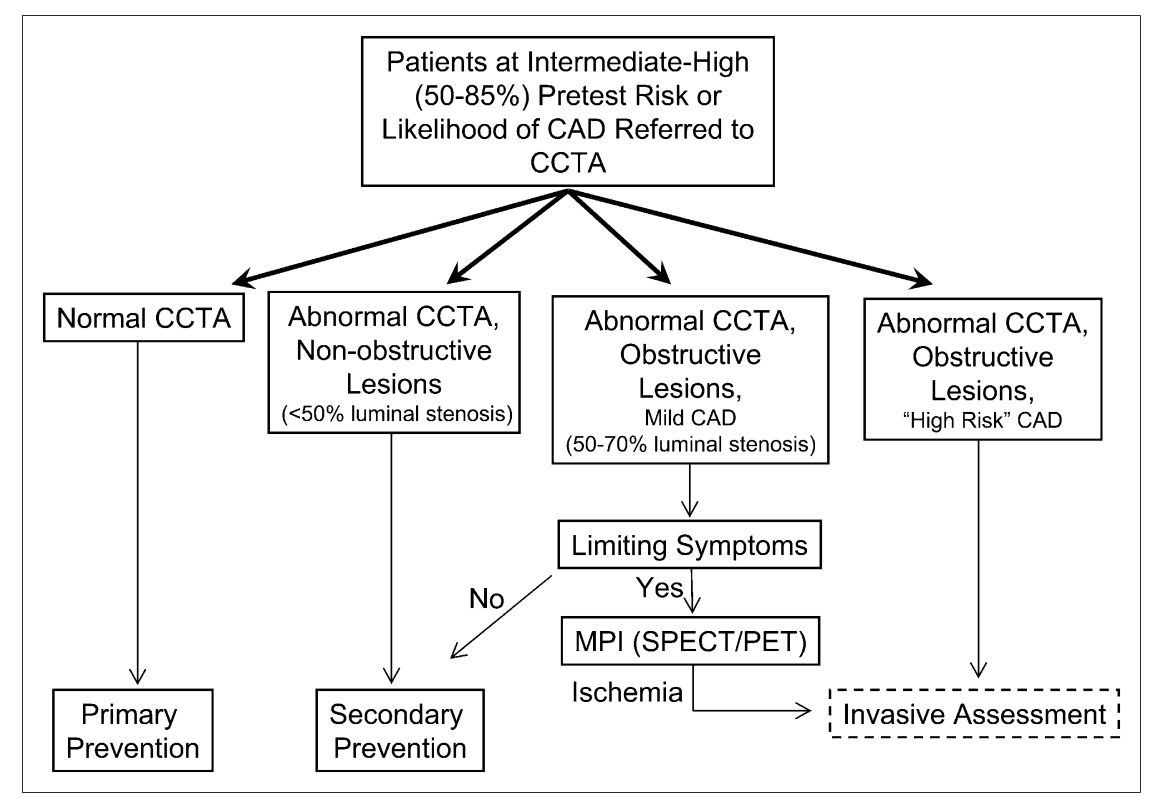

endpoint (death, myocardial infarction, repeated PCI, and coronary artery bypass grafting) by $30 \%(P=0.02)$. Further, the rate of mortality and myocardial infarction at $1 \mathrm{y}$ was reduced by $34 \%(P=0.04)$. Thus, a strategy of physiologic assessment of coronary lesions before revascularization may identify a subgroup of patients who may benefit from revascularization. However, fractional flow reserve measurements are not routinely used across all centers performing PCI before revascularization.

This approach can be extended to the use of an MPIbased decision-making approach in identifying candidates for revascularization versus medical therapy. Hachamovitch et al. demonstrated a survival benefit with medical therapy for patients showing mild or no ischemia on MPI, compared with similar patients who underwent revascularization; however, revascularization produced a survival benefit over medical therapy in patients with moderate to severe ischemia (20). Similarly, in a subsequent study, these authors found that the relative benefits of revascularization over medical therapy were seen for ischemia but not for ejection fraction (21). These results were extended to asymptomatic diabetic patients in a subsequent study from the Mayo Clinic group that again found the presence of inducible ischemia to be associated with improved survival in the setting of revascularization (22). In contrast, Tarakji et al., from the Cleveland Clinic, found no such survival benefit using a stress PET and ${ }^{18} \mathrm{~F}-\mathrm{FDG}$ protocol in a cohort of patients with advanced CAD; however, these patients had more severe and extensive CAD than patients in previous studies (23). Indeed, in a recent study, the mortality benefit of a perfusion-guided approach was found to also apply to patients with a previous history of CAD as long as significant scarring $(>10 \%$ of myocardium) was absent (24).

Although the COURAGE study called into question the use of a revascularization strategy, assignment of patients to either revascularization or medical therapy in that study was not based on the extent of ischemia. The COURAGE nuclear substudy compared optimal medical therapy, versus revascularization plus optimal medical therapy, in 314 patients who underwent noninvasive MPI before and 6-18 mo after randomization (25). That substudy demonstrated that, compared with optimal medical therapy alone, revascularization in addition to optimal medical therapy led to decreased ischemia with an associated decrease in adverse cardiac events. The above-mentioned studies suggest that knowledge of the extent of ischemia may be valuable in guiding clinical management by allowing us to identify the treatment strategy-medical versus revascularization - that would confer a mortality benefit.

\section{PHYSIOLOGY-BASED APPROACH: MPI}

\section{Prognostic Value}

An extensive body of literature supports the ability of stress MPI to successfully risk-stratify a variety of patient groups (26). A normal exercise MPI result in patients who are able to attain more than $85 \%$ of maximal predicted heart rate, or a normal pharmacologic stress MPI result, can identify patients at low risk for future cardiac events (27). A metaanalysis of 19 studies ( $n=39,173$ patients) found that a normal- or low-risk stress MPI result was associated with an annual cardiac event rate of only $0.6 \%$ (25th-75th percentiles, $0.5 \%-0.9 \%$ ) (28). Even in the presence of angiographically documented CAD, a normal stress MPI result still defines a group with only a $1 \%$ annual risk of cardiac events (29). On the other hand, perfusion abnormalities of increasing extent and severity are closely associated with increasing patient risk (26). These findings have been shown in multiple patient subgroups, using multiple radioisotopes, as well as several different stress agents.

Although this risk-based approach to the use of cardiovascular imaging has dominated thinking for several years, 
basing patient management on the results of risk stratification in no way ensures improvement and patient well-being or survival. In light of increasing calls for a value-based health care system, we will need to demonstrate that patients can benefit from the use of these expensive imaging modalities. Thus, despite the past emphasis on risk-based testing, a newer paradigm of benefit-based testing is slowly emerging (26).

\section{SPECT MPI}

A post-SPECT MPI patient management strategy focusing on the identification of potential revascularization candidates is shown in Figure 2. Patients with abnormal SPECT studies showing more than $10 \%-15 \%$ of the myocardium ischemic are candidates for referral for invasive assessment with an eye to possible revascularization. On the other hand, patients whose SPECT result is normal, abnormal but with slight ischemia $(<10 \%-15 \%$ of myocardium), or abnormal but with no inducible ischemia are not candidates for catheterization and would be considered for preventive treatment only. The exception to this approach in these 3 groups of patients is when underestimation of ischemia is a concern. Some patients with angiographically significant CAD may manifest a normal SPECT result because of balanced ischemia due to the relative nature of perfusion defect assessment. When there is an equivocal SPECT result, a normal SPECT result but recurrent or limiting symptoms, or discordance between clinical findings during stress and findings on stress SPECT, CT coronary angiography may be appropriate (1). In the subgroup of patients with significant stress-induced ischemic electrocardiography changes, stress-induced symptoms, serious stress-induced ventricular arrhythmias, and unequivocal transient ischemic dilation or other ancillary SPECT findings, there may be reasonable grounds for referral for catheterization despite the absence of extensive SPECT-identified ischemia. Finally, on the basis of the data presented here, patients with scarring of more than $10 \%-15 \%$ of the myocardium may not be candidates for revascularization and may therefore be candidates for a preventive strategy.

\section{PET MPI}

Compared with SPECT MPI, PET MPI has several distinct advantages. First, the increased sensitivity of this technique, along with superior spatial and temporal resolution, provides better diagnostic accuracy $(2,12,30)$. Further, because stress images are obtained during infusion of a vasodilator, the results of stress gated PET represent peak stress wall motion. Although vasodilator-stress gated images are not the equivalent of exercise gated images, investigators have found that the failure to show an increased ejection fraction on stress gated PET images compared with rest images is strongly associated with the presence of extensive CAD (31). This ejection fraction reserve has also been shown to be prognostically significant (32). Finally, the ability to quantify absolute myocardial blood flow $(2,33,34)$ with stress PET MPI permits identification of patients in whom the relative regional distribution of tracer may appear normal because of a balanced reduction of blood flow.

The ability to quantify myocardial blood flow with ${ }^{82} \mathrm{Rb}$ PET has been shown to be useful in identifying 3-vessel CAD (35). Furthermore, mean flow rate has been shown to serve as a useful prognostic marker for adverse cardiac events. Herzog et al. demonstrated that in the setting of a normal MPI result, a preserved mean flow rate of more than 2.0 may provide a warranty period of $3 \mathrm{y}$ versus a reduced mean flow rate (36). Also, mean flow rate results were found to stratify patients-both those with normal and those with abnormal stress perfusion-with respect to their risk of adverse cardiovascular events. PET MPI in combination with quantification of regional myocardial blood flow or regional

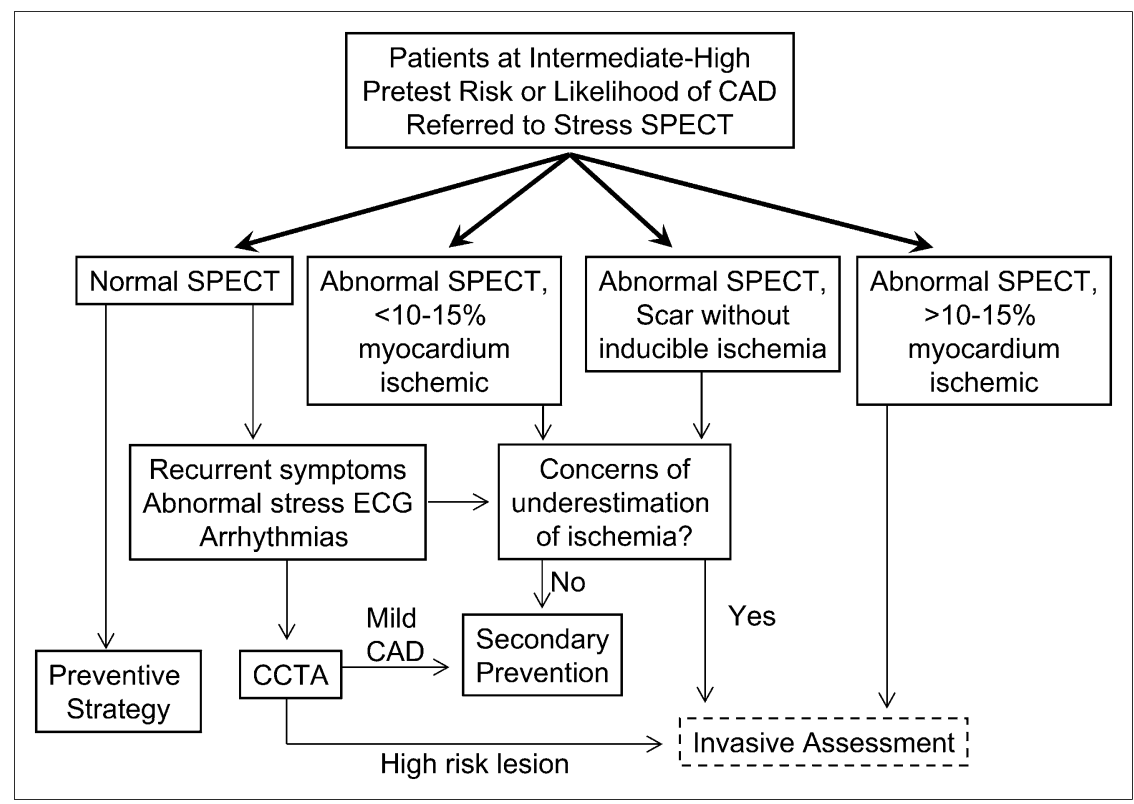

FIGURE 2. Potential testing algorithm based on use of stress cardiac SPECT MPI. 
mean flow rate may therefore improve our ability to identify patients who might benefit from revascularization and may provide better prognostic estimates of future cardiac events. Although mean flow rate measurements may be obtainable from PET-based perfusion imaging, potential sources of errors in measurement, including the effect of high driving pressure and high resting flow rates, are still being characterized. Furthermore, still unexplored is how mean flow rate may differentiate between patients who have epicardial stenoses and patients who have abnormalities with subendocardial microvascular perfusion.

Figure 3 outlines a potential post-PET MPI patient management approach based on the use of ejection fraction reserve and flow quantification. Patients with a normal PET result can be referred directly for a preventive strategy, because normal is defined on the basis of perfusion, ejection fraction reserve, and coronary flow reserve. Patients with abnormal PET perfusion and extensive myocardial ischemia ( $>10 \%-15 \%$ of myocardium) are potential candidates for revascularization and would be referred for invasive assessment. The remaining patients, those with slight inducible ischemia or those with slight scarring without inducible ischemia, would be referred for a preventive strategy unless there is other evidence of extensive ischemia, such as a compromised ejection fraction reserve or a significant global reduction in coronary flow reserve. It is likely that patients with extensive scarring may not be eligible for revascularization. To date, there have been no published studies demonstrating a survival benefit with revascularization on the basis of PET results. However, such a benefit has been shown with SPECT MPI and is likely to exist with PET MPI as well.

\section{HYBRID APPROACH}

Recently, an attempt has been made to perform physiologic and anatomic imaging simultaneously with a hybrid PET MPI/CT coronary angiography technique (37). In their study of 104 patients who underwent both the hybrid technique and invasive coronary angiography, Kajander et al. found that, using invasive coronary angiography as the gold standard, the hybrid technique had a higher positive predictive value than either technique alone ( $81 \%$ for CT, $86 \%$ for PET, and $100 \%$ for the hybrid technique) and a similarly higher specificity $(87 \%, 91 \%$, and $100 \%$, respectively). That study also showed that an absolute myocardial blood flow of less than $2.5 \mathrm{~mL} / \mathrm{min} / \mathrm{g}$ was the optimal cutoff between normal and abnormal perfusion and that a myocardial blood flow of less than $2 \mathrm{~mL} / \mathrm{min} / \mathrm{g}$ was unequivocally abnormal. The mean radiation dose in most of their patients was $9.3 \mathrm{mSv}$ when prospective triggering was used, and this value was comparable to a mean estimated radiation dose of $7 \mathrm{mSv}$ for invasive angiography. Thus, further advances in technology may enable simultaneous assessment of both anatomy and physiology, resulting in an even better diagnostic modality with the added benefit of providing prognostic information. The major limitation of the hybrid technique is not only radiation exposure but also increased cost. Before widespread acceptance, it will be central to identify in which patient subsets testing strategies can be enhanced by the use of this hybrid technique.

\section{SEQUENTIAL APPROACH}

Finally, it is possible that these 2 technologies are best used by integrating anatomy- and physiology-based imaging in the appropriate setting, thereby maximally utilizing the strengths of each. With a high negative predictive value, CT coronary
FIGURE 3. Potential testing algorithm based on use of stress cardiac PET MPI. $\mathrm{EF}=$ ejection fraction; CFR = coronary flow reserve.

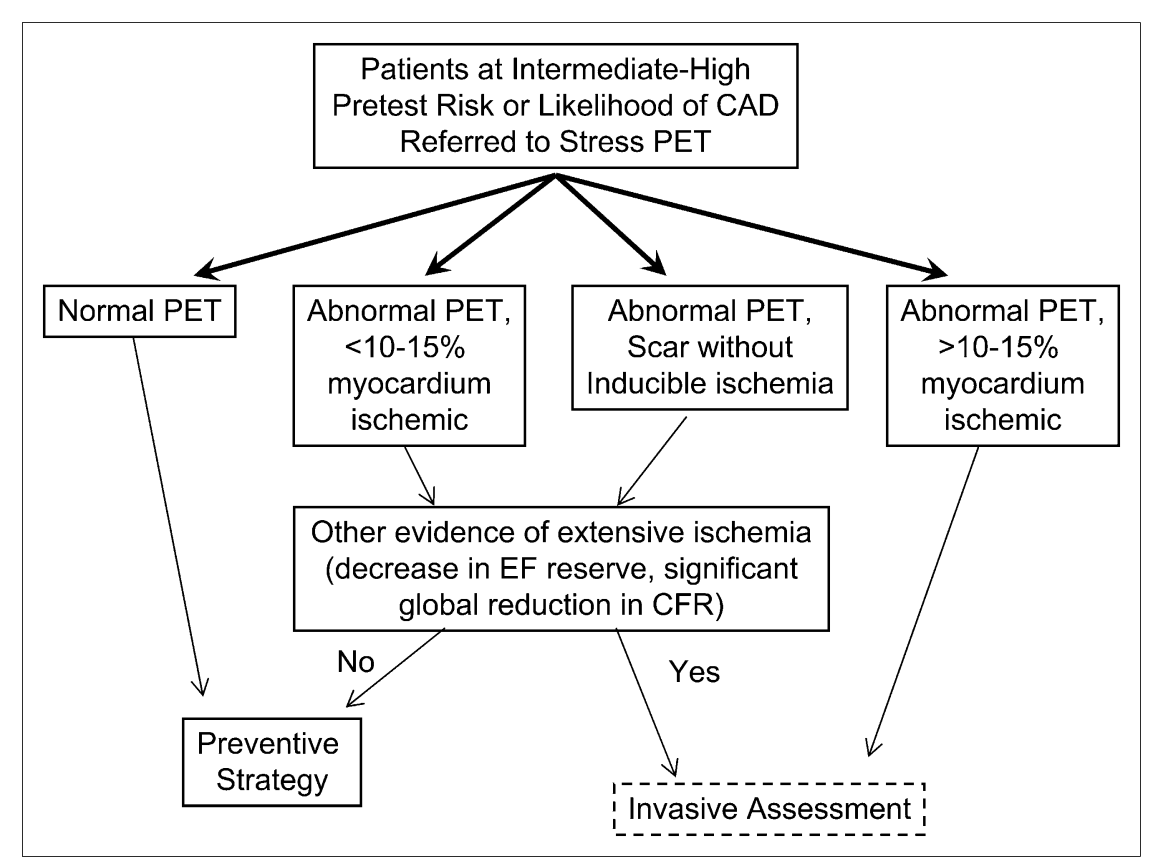


angiography qualifies as an excellent initial test to exclude the presence of CAD. On the other hand, the quantification of inducible ischemia by MPI enhances our ability to identify the optimal therapeutic approach: medical therapy versus possible revascularization. Regardless of which diagnostic test is chosen first, an equivocal or a borderline result may be investigated with a complementary imaging test (1). As shown in Figure 1, patients with a normal result on CT coronary angiography are candidates for primary prevention whereas those with an abnormal result and nonobstructive or limited CAD are candidates for secondary prevention. The remaining patients, who have mild CAD with limiting symptoms or functional capacity, would be candidates for MPI. The advantages of this approach include minimization of radiation exposure, minimization of missed diagnoses due to balanced reduction (because of the negative predictive value of CT coronary angiography), and reduction of excess catheterization and revascularization due to false-positive CT coronary angiography studies (prerevascularization documentation of ischemic burden). Similarly, as outlined in Figure 2, CT coronary angiography may have an important role to play in patients with discordance between clinical response to stress and MPI (1).

\section{CONCLUSION}

Both anatomy- and physiology-based approaches to patient management have advantages and limitations. Compared with the latter, the former has a superior ability to exclude disease and does not miss high-risk CAD. However, it is limited by the possibility of overestimating the severity of CAD and of potentially failing to determine which posttest therapeutic approach optimizes treatment benefit. On the other hand, although a physiology-based approach could potentially identify optimal therapeutic strategies, the possibility of both false-positive and false-negative findings is a concern. It is possible that the optimal approach in stable, symptomatic, intermediate-risk patients may consist of the use of CT coronary angiography as the initial test, with those patients found to have significant obstructive disease referred for MPI. Alternatively, PET MPI, by combining perfusion imaging, left ventricle functional assessment, and coronary flow reserve, may eventually achieve primary status as a single-modality approach. Extensive studies are necessary to ascertain both the performance characteristics of these modalities and their relative cost effectiveness as part of testing strategies.

\section{REFERENCES}

1. Berman DS, Hachamovitch R, Shaw LJ, et al. Roles of nuclear cardiology, cardiac computed tomography, and cardiac magnetic resonance: assessment of patients with suspected coronary artery disease. J Nucl Med. 2006;47:74-82.

2. Di Carli MF, Hachamovitch R. New technology for noninvasive evaluation of coronary artery disease. Circulation. 2007;115:1464-1480.

3. Budoff MJ, Dowe D, Jollis JG, et al. Diagnostic performance of 64-multidetector row coronary computed tomographic angiography for evaluation of coronary artery stenosis in individuals without known coronary artery disease: results from the prospective multicenter ACCURACY (Assessment by Coronary Computed
Tomographic Angiography of Individuals Undergoing Invasive Coronary Angiography) trial. J Am Coll Cardiol. 2008;52:1724-1732.

4. Di Carli MF, Dorbala S, Curillova Z, et al. Relationship between CT coronary angiography and stress perfusion imaging in patients with suspected ischemic heart disease assessed by integrated PET-CT imaging. J Nucl Cardiol. 2007;14:799-809.

5. Gaemperli O, Schepis T, Koepfli P, et al. Accuracy of 64-slice CT angiography for the detection of functionally relevant coronary stenoses as assessed with myocardial perfusion SPECT. Eur J Nucl Med Mol Imaging. 2007;34:1162-1171.

6. Gaemperli O, Schepis T, Valenta I, et al. Functionally relevant coronary artery disease: comparison of 64-section CT angiography with myocardial perfusion SPECT. Radiology. 2008;248:414-423.

7. Schuijf JD, Wijns W, Jukema JW, et al. Relationship between noninvasive coronary angiography with multi-slice computed tomography and myocardial perfusion imaging. J Am Coll Cardiol. 2006;48:2508-2514.

8. Tamarappoo BK, Gutstein A, Cheng VY, et al. Assessment of the relationship between stenosis severity and distribution of coronary artery stenoses on multislice computed tomographic angiography and myocardial ischemia detected by single photon emission computed tomography. J Nucl Cardiol. 2010;17:791-802.

9. Hulten EA, Carbonaro S, Petrillo SP, Mitchell JD, Villines TC. Prognostic value of cardiac computed tomography angiography: a systematic review and metaanalysis. J Am Coll Cardiol. 2011;57:1237-1247.

10. Min JK, Shaw LJ, Devereux RB, et al. Prognostic value of multidetector coronary computed tomographic angiography for prediction of all-cause mortality. J Am Coll Cardiol. 2007;50:1161-1170.

11. van Werkhoven JM, Schuijf JD, Gaemperli O, et al. Prognostic value of multislice computed tomography and gated single-photon emission computed tomography in patients with suspected coronary artery disease. J Am Coll Cardiol. 2009;53:623-632.

12. Di Carli MF, Dorbala S, Meserve J, El Fakhri G, Sitek A, Moore SC. Clinical myocardial perfusion PET/CT. J Nucl Med. 2007;48:783-793.

13. Boden WE, O'Rourke RA, Teo KK, et al. Optimal medical therapy with or without PCI for stable coronary disease. N Engl J Med. 2007;356:1503-1516.

14. Kern MJ, Lerman A, Bech JW, et al. Physiological assessment of coronary artery disease in the cardiac catheterization laboratory: a scientific statement from the American Heart Association Committee on Diagnostic and Interventional Cardiac Catheterization, Council on Clinical Cardiology. Circulation. 2006;114:1321-1341.

15. Bech GJ, De Bruyne B, Pijls NH, et al. Fractional flow reserve to determine the appropriateness of angioplasty in moderate coronary stenosis: a randomized trial. Circulation. 2001;103:2928-2934.

16. Pijls NH, van Schaardenburgh P, Manoharan G, et al. Percutaneous coronary intervention of functionally nonsignificant stenosis: 5-year follow-up of the DEFER Study. J Am Coll Cardiol. 2007;49:2105-2111.

17. Bech GJ, Droste H, Pijls NH, et al. Value of fractional flow reserve in making decisions about bypass surgery for equivocal left main coronary artery disease. Heart. 2001;86:547-552.

18. Chamuleau SA, Meuwissen M, Koch KT, et al. Usefulness of fractional flow reserve for risk stratification of patients with multivessel coronary artery disease and an intermediate stenosis. Am J Cardiol. 2002;89:377-380.

19. Tonino PA, De Bruyne B, Pijls NH, et al. Fractional flow reserve versus angiography for guiding percutaneous coronary intervention. N Engl J Med. 2009;360: 213-224.

20. Hachamovitch R, Hayes SW, Friedman JD, Cohen I, Berman DS. Comparison of the short-term survival benefit associated with revascularization compared with medical therapy in patients with no prior coronary artery disease undergoing stress myocardial perfusion single photon emission computed tomography. Circulation. 2003;107:2900-2907.

21. Hachamovitch R, Di Carli MF. Nuclear cardiology will remain the "gatekeeper" over CT angiography. J Nucl Cardiol. 2007;14:634-644.

22. Sorajja P, Chareonthaitawee $P$, Rajagopalan N, et al. Improved survival in asymptomatic diabetic patients with high-risk SPECT imaging treated with coronary artery bypass grafting. Circulation. 2005;112:I311-I316.

23. Tarakji KG, Brunken R, McCarthy PM, et al. Myocardial viability testing and the effect of early intervention in patients with advanced left ventricular systolic dysfunction. Circulation. 2006;113:230-237.

24. Hachamovitch R, Rozanski A, Shaw LJ, et al. Impact of ischaemia and scar on the therapeutic benefit derived from myocardial revascularization vs. medical therapy among patients undergoing stress-rest myocardial perfusion scintigraphy. Eur Heart J. 2011;32:1012-1024.

25. Shaw LJ, Berman DS, Maron DJ, et al. Optimal medical therapy with or without percutaneous coronary intervention to reduce ischemic burden: results from the Clinical Outcomes Utilizing Revascularization and Aggressive Drug Evaluation (COURAGE) trial nuclear substudy. Circulation. 2008;117:1283-1291.

26. Berman DS, Shaw LJ, Hachamovitch R, et al. Comparative use of radionuclide stress testing, coronary artery calcium scanning, and noninvasive coronary angiography for diagnostic and prognostic cardiac assessment. Semin Nucl Med. 2007;37:2-16. 
27. Klocke FJ, Baird MG, Lorell BH, et al. ACC/AHA/ASNC guidelines for the clinical use of cardiac radionuclide imaging: executive summary - a report of the American College of Cardiology/American Heart Association Task Force on Practice Guidelines (ACC/AHA/ASNC Committee to Revise the 1995 Guidelines for the Clinical Use of Cardiac Radionuclide Imaging). Circulation. 2003;108:1404-1418.

28. Shaw LJ, Iskandrian AE. Prognostic value of gated myocardial perfusion SPECT. J Nucl Cardiol. 2004;11:171-185.

29. Gibbons RJ, Smith SC Jr, Antman E. American College of Cardiology/American Heart Association clinical practice guidelines: Part II: evolutionary changes in a continuous quality improvement project. Circulation. 2003;107:3101-3107.

30. Schwaiger M, Ziegler S, Nekolla SG. PET/CT: challenge for nuclear cardiology. J Nucl Med. 2005;46:1664-1678.

31. Dorbala S, Vangala D, Sampson U, Limaye A, Kwong R, Di Carli MF. Value of vasodilator left ventricular ejection fraction reserve in evaluating the magnitude of myocardium at risk and the extent of angiographic coronary artery disease: a ${ }^{82}$ Rb PET/CT study. J Nucl Med. 2007;48:349-358.
32. Dorbala S, Hachamovitch R, Curillova Z, et al. Incremental prognostic value of gated $\mathrm{Rb}-82$ positron emission tomography myocardial perfusion imaging over clinical variables and rest LVEF. JACC Cardiovasc Imaging. 2009;2:846-854.

33. Sampson UK, Dorbala S, Limaye A, Kwong R, Di Carli MF. Diagnostic accuracy of rubidium- 82 myocardial perfusion imaging with hybrid positron emission tomography/computed tomography in the detection of coronary artery disease. J Am Coll Cardiol. 2007;49:1052-1058.

34. Schelbert HR. Blood flow and metabolism by PET. Cardiol Clin. 1994;12:303-315.

35. Parkash R, deKemp RA, Ruddy TD, et al. Potential utility of rubidium 82 PET quantification in patients with 3 -vessel coronary artery disease. $\mathrm{J} \mathrm{Nucl} \mathrm{Cardiol}$. 2004;11:440-449.

36. Herzog BA, Husmann L, Valenta I, et al. Long-term prognostic value of ${ }^{13} \mathrm{~N}$ ammonia myocardial perfusion positron emission tomography added value of coronary flow reserve. J Am Coll Cardiol. 2009;54:150-156.

37. Kajander S, Joutsiniemi E, Saraste M, et al. Cardiac positron emission tomography/computed tomography imaging accurately detects anatomically and functionally significant coronary artery disease. Circulation. 2010;122:603-613. 